\title{
HIV-IG pharmacokinetics in HIV-infected pregnant women
}

The 'short-term safety' of anti-HIV-immune globulin* (HIV-IG) in pregnant women with HIV infection has been demonstrated in a double-blind, randomised study, reports the Pediatric AIDS Clinical Trials Group (ACTG) Protocol 185 Pharmacokinetic Study Group. HIV-IG was well tolerated by the women and their neonates, say the researchers. However, the long-term effects of HIV-IG in this indication remain unknown.

\section{Study protocol}

The efficacy of HIV-IG for the prevention of maternal-infant HIV transmission was evaluated in 28 women between 20 and 30 weeks' gestation, whose CD $4+$ counts were $\leq 500$ cells $/ \mathrm{mm}^{3}$. The women were receiving zidovudine and they were randomised to start intravenous HIV-IG $(n=12)$ or standard intravenous immune globulin (IVIG; 16) $200 \mathrm{mg} / \mathrm{kg}$, every 28 days until delivery.

Within 12 hours after birth, the 26 evaluable neonates received a single infusion of HIV-IG or IVIG $200 \mathrm{mg} / \mathrm{kg}$ in accordance with the maternal regimen.

\section{Pharmacokinetics revealed}

Trough p24 antibody levels appeared to rise with successive HIV-IG administrations in the women, and sustained p24 antibody elevation was observed at 28 days postpartum in HIV-IG, but not IVIG, recipients. HIV-IG administration was associated with substantial elevations in serum titres of p24 antibody in women and neonates.

The data in this phase I study (conducted within the ACTG protocol 185 study) reveal that HIV-IG pharmacokinetics are best described by a 2-compartment model. Typical values for the women's HIV-IG disposition during the first 2 infusions were a half-life of approximately 15 days, a volume of distribution of approximately $70 \mathrm{ml} / \mathrm{kg}$ and a clearance rate of 4 $\mathrm{ml} / \mathrm{kg} /$ day. The volume of distribution and half-life increased after 3 infusions. Neonatal HIV-IG disposition showed biexponential decay with a mean half-life of 30 days and a clearance rate of $4 \mathrm{ml} / \mathrm{kg} /$ day.

\section{* NABI; phase III}

Lambert JS, Mofenson LM, Fletcher CV, Pediatric AIDS Clinical Trials Group Protocol 185 Pharmacokinetic Study Group. Safety and pharmacokinetics of hyperimmune anti-human immunodeficiency virus (HIV) immunoglobulin administered to HIV-infected pregnant women and their newborns. Journal of Infectious Diseases 175: 283-291, Feb 1997

Editorial comment: HIV-IG has orphan drug status in the US for use in pregnant women with HIV infection. 